\title{
ATF3 deficiency impairs the proliferative-secretory phase transition and decidualization in RIF patients
}

\author{
Zhilong Wang $\mathbb{D}^{1}$, Yang Liu', Jingyu Liu', Na Kong', Yue Jiang', Ruiwei Jiang $\mathbb{D}^{1}$, Xin Zhen', Jidong Zhou',

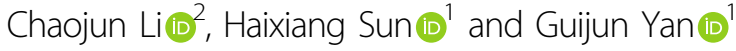

\begin{abstract}
Decidualization is a complex process involving cellular proliferation and differentiation of the endometrial stroma and is required to establish and support pregnancy. Dysregulated decidualization has been reported to be a critical cause of recurrent implantation failure (RIF). In this study, we found that Activating transcription factor 3 (ATF3) expression was significantly downregulated in the endometrium of RIF patients. Knockdown of ATF3 in human endometrium stromal cells (hESCs) hampers decidualization, while overexpression could trigger the expression of decidual marker genes, and ameliorate the decidualization of hESCs from RIF patients. Mechanistically, ATF3 promotes decidualization by upregulating FOXO1 via suppressing miR-135b expression. In addition, the endometrium of RIF patients was hyperproliferative, while overexpression of ATF3 inhibited the proliferation of hESCs through CDKN1A. These data demonstrate the critical roles of endometrial ATF3 in regulating decidualization and proliferation, and dysregulation of ATF3 in the endometrium may be a novel cause of RIF and therefore represent a potential therapeutic target for RIF.
\end{abstract}

\section{Introduction}

Reciprocal intimate cross-talk between the activated blastocyst and the receptive uterus is essential for successful implantation, and therefore, for pregnancy outcome $^{1}$. The desire to have children is powerful and widespread, however, about $15 \%$ of couples are childless because of infertility, which is a worldwide social and economic concern ${ }^{2}$. Currently, due to impressive advances in assisted reproductive technology (ART) and, in particular, the advent of more effective embryo selection and cryopreservation, many underlying causes of human infertility have been overcome; however, there are still many patients who continue to experience ART failure after serial IVF attempts ${ }^{3}$, probably owing to embryos being transferred into a nonreceptive uterus. Recurrent

\footnotetext{
Correspondence: Haixiang Sun (stevensunz@163.com) or

Guijun Yan (yanguijun55@163.com)

${ }^{1}$ Reproductive Medicine Center, The Affiliated Drum Tower Hospital of Nanjing University Medical School, 210008 Nanjing, People's Republic of China

${ }^{2}$ Nanjing University Medical School, 210008 Nanjing, People's Republic of

China

These authors contributed: Zhilong Wang, Yang Liu, Jingyu Liu

Edited by $Y$. Shi
}

implantation failure (RIF) is one of the major causes of infertility in ART programs, and these pregnancy failures are believed to be mainly due to defects in early pregnancy events, including implantation and decidualization ${ }^{4}$.

In recent years, it has become apparent that endometrial factors have an important role in implantation ${ }^{5,6}$. The human endometrial cycle can be divided into 2 phases: the proliferative phase, which is marked by the active growth of stromal, epithelial, and vascular cells, and the secretory phase, during which the endometrium undergoes decidualization to prepare for implantation ${ }^{7,8}$. The proper transition between the proliferative and secretory phases is a prerequisite for the establishment of the WOI and the subsequent pregnancy. Decidualization deficiency or abnormality may be one of the major maternal causes of RIF $^{9,10}$. Besides, the receptivity window of RIF patients was delayed by $1-3$ days ${ }^{11,12}$, suggesting that the acquisition of the endometrial receptivity is slower in RIF patients. Nevertheless, the critical underlying molecular mechanisms remain largely unknown.

Activating transcription factor 3 (ATF3) belongs to the ATF/CREB transcription factor family, which binds to the

\section{(c) The Author(s) 2021}

(c) (i) Open Access This article is licensed under a Creative Commons Attribution 4.0 International License, which permits use, sharing, adaptation, distribution and reproduction cc) in any medium or format, as long as you give appropriate credit to the original author(s) and the source, provide a link to the Creative Commons license, and indicate if changes were made. The images or other third party material in this article are included in the article's Creative Commons license, unless indicated otherwise in a credit line to the material. If material is not included in the article's Creative Commons license and your intended use is not permitted by statutory regulation or exceeds the permitted use, you will need to obtain permission directly from the copyright holder. To view a copy of this license, visit http://creativecommons.org/licenses/by/4.0/. 
consensus ATF/cAMP response element in numerous promoters ${ }^{13,14}$. It can also regulate various biological functions independent of its transcriptional activity ${ }^{15,16}$. ATF3 acts as a hub of the cellular adaptive-response network and has vital roles in modulating metabolism, immunity, the cell cycle, and differentiation. Distinct from other ATF/CREB proteins, ATF3 expression is rapidly induced by a wide range of extracellular signals, such as nutrient deprivation, endoplasmic reticulum stress, cytokines, chemokines, and hormones ${ }^{17-19}$. However, little is known about whether uterine ATF3 has any role in decidualization, the proliferative-secretory phase transition of the menstrual cycle, and the pathological relationship with RIF. In this study, we describe a novel mechanism of dysregulation of ATF3 in the endometrium from RIF patients hampers decidualization that may be a new cause of RIF.

\section{Results}

\section{ATF3 is deficient in the endometrium of RIF patients}

In our previous study, we demonstrated that the endometrium of RIF patients displays a distinct transcriptional profile and suggests that dysregulation of decidualization may be a cause of implantation failure ${ }^{10}$. Through RNA-seq analysis, we found that ATF3 expression was significantly downregulated in the endometrium of RIF women (Fig. 1A, B), while other members of ATF/ CREB did not change significantly (Supplemental Fig. S1). qPCR was then used to validate that in the mid-secretory phase, subjects with RIF had a 0.5-fold lower ATF3 expression level than controls (Fig. 1C), consistent with the western blot results (Fig. 1D, E). As shown in Fig. 1F, G, the expression of ATF3 in the endometrial stromal cells from RIF patients was significantly decreased, as determined by immunohistochemical staining. Through analysis of publicly accessible data banks, we found that the placenta exhibits a high level of ATF3 expression, whereas its level ranges from medium to high in the uterus and endometrium, indicating an important role for ATF3 in human endometrial cycling (Supplemental Fig. S1). We, therefore, wondered whether an element of the infertility phenotype could be caused by an alteration in the expression of ATF3. In addition, we found that the expression of the decidualization marker PRL was decreased in the endometrium from RIF patients (Fig. $1 \mathrm{H})$.

\section{Spatiotemporal expression of ATF3 in the human endometrium}

To address the pathophysiological significance of ATF3 during early pregnancy, we then analyzed the ATF3 expression pattern in the human endometrium by immunohistochemical staining. The results show that the ATF3 protein abundance was higher in the mid-secretory phase than in the proliferative phase of the endometrium, especially in the endometrial stromal cells (Fig. 2A, B). We also analyzed the relative expression of temporal ATF3 expression across the cycle from a public dataset (Fig. 2C). Additionally, a recent single-cell RNA-seq analysis suggests that ATF3 may be a probable driver for decidualization ${ }^{20}$, and using this data we found that ATF3 is elevated in the stromal cells since the early-secretory phase (Fig. 2D). Besides, the expression of ATF3 in all cells of the endometrium is upregulated in the midsecretory compared with the early-secretory phase (Fig. 2E).

We also examined the levels of ATF3 and other members of the ATF/CREB family in hESCs treated with 8-BrcAMP plus MPA for $0.5-16 \mathrm{~h}$ using qPCR. The results reveal that ATF3 was rapidly elevated by $\sim 40$-fold at $\sim 1 \mathrm{~h}$ in hESCs (Fig. 2F), while other ATFs showed no significant change (Supplemental Fig. S2). Western blot analysis also confirmed that the expression of ATF3 was increased after in vitro decidualization (Fig. 2G). Gene set enrichment analysis (GSEA) results showed that the ATF3 target term was significantly upregulated during decidualization $(\mathrm{NES}=1.436, P<0.01)$ (Fig. $2 \mathrm{H}$ ). The results indicate that decidualization stimuli could upregulate the expression of ATF3 in the stroma of the endometrium.

\section{Lack of ATF3 impairs decidualization}

Various functions have been ascribed to decidual prolactin (PRL) which is now established as a widely used marker to assess the differentiation status of hESCs in culture. As shown in Supplemental Fig. S3A, the expression of ATF3 showed a positive relationship with PRL in the endometrium. When hESCs were transfected with small interfering RNA specific for ATF3 for $48 \mathrm{~h}$ followed by treatment with 8 -Br-cAMP and MPA for 3 days, the expression and secretion of PRL were substantially decreased (Fig. 2I, J). We further examined the effect of ATF3 on the reorganization of the cytoskeleton, which reflects a morphological marker of decidualization ${ }^{21}$. As shown in Fig. 2K, decidualized hESCs displayed polygonal cell morphologies with a random distribution of F-actin filaments. When knockdown of endogenous ATF3, stromal cells maintained a fibroblast-like phenotype under decidualization stimuli. Furthermore, adenovirusmediated overexpression of ATF3 in hESCs markedly increased the expression and secretion of PRL in the absence of exogenous hormones (Supplemental Fig. S3B, C). In addition, ATF3 overexpression resulted in a noticeable transformation from a long fibroblast-like shape into a rounder shape, and the actin filaments were randomly arranged (Supplemental Fig. S3D). Together, these results suggest that ATF3 may be functionally important for the regulation of decidualization. 


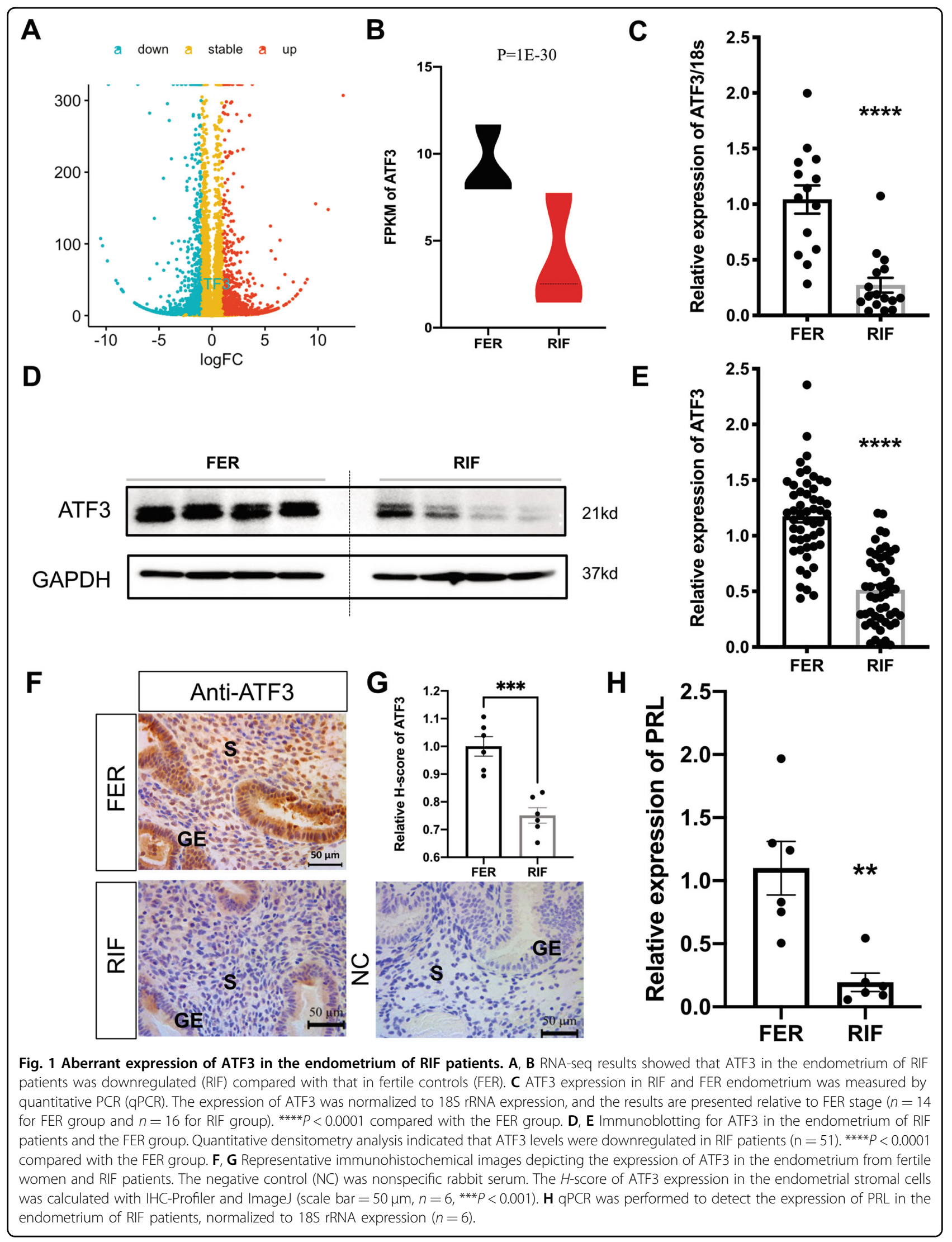




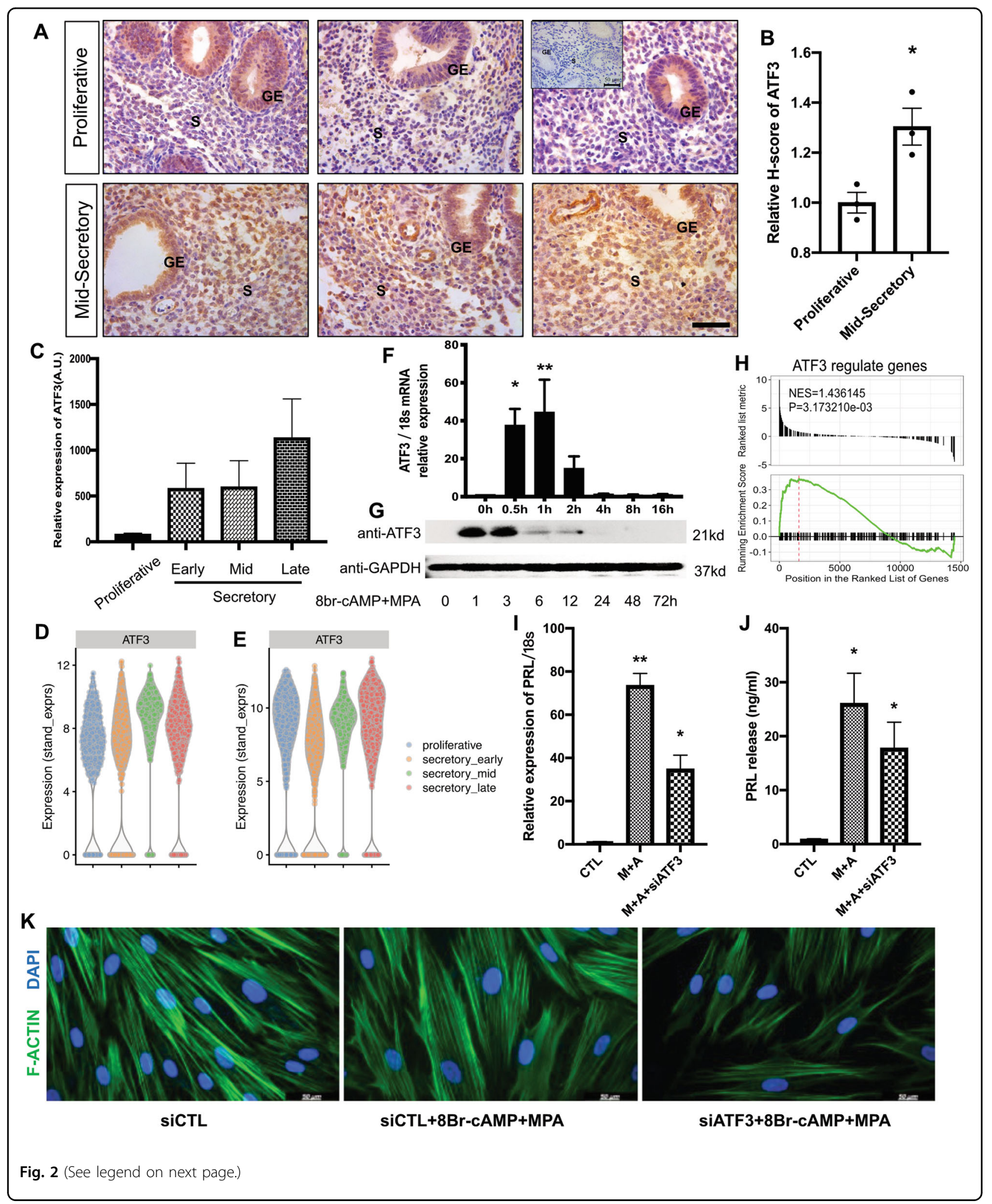


(see figure on previous page)

Fig. 2 The temporal expression and function of ATF3 in the endometrium. A, B Immunohistochemistry analysis with an anti-ATF3 antibody. Proliferative and mid-secretory phase endometrial tissue samples from normal fertile women. The negative control (NC) was nonspecific rabbit serum. The H-score of ATF3 expression in the endometrial stromal cells was calculated with IHC-Profiler and ImageJ (Scale bar $=50 \mu \mathrm{m}, n=3,{ }^{*} P<$ 0.05). C Expression of ATF3 in proliferative and early-, mid-, and late-luteal phase endometrium. Each bar represents an individual biopsy. The data were retrieved from microarray data deposited in the Gene Expression Omnibus (GDS2052). D, E ATF3 expression pattern analyzed with a recent single-cell RNA-seq analysis in stromal cells (D) and all cells (E). The data were retrieved from microarray data deposited in the Gene Expression Omnibus (GSE111976). F The expression pattern of ATF3 in hESCs treated with $0.5 \mathrm{mM} 8$ 8-Br-CAMP and $1 \mu \mathrm{M} M \mathrm{MPA}(\mathrm{M}+\mathrm{A}$ ) for different periods of time $(0,0.5,1,2,4,8$, or $16 \mathrm{~h})$ was evaluated by $q \mathrm{PCR} .{ }^{*} P<0.05,{ }^{* *} P<0.01$. G The expression pattern of ATF3 in hESCs treated with $0.5 \mathrm{mM} 8-\mathrm{Br}-\mathrm{CAMP}$ and $1 \mu \mathrm{M} \mathrm{MPA}(\mathrm{M}+\mathrm{A})$ for different periods of time $(0,1,3,6,12,24$, 48, or $72 \mathrm{~h})$ was evaluated by western blot. $\mathbf{H}$ GSEA results showed that the ATF3regulated gene term was enriched during in vitro decidualization. I, $\mathbf{J}$ hESCs were transfected with siATF3 or siCtl for $48 \mathrm{~h}$ and then treated with a decidualization stimulus. The expression and secretion of PRL were measured by qPCR and ELISA, respectively. ${ }^{*} P<0.05$, ${ }^{* *} P<0.01$ compared with the CTL/M + A group (8-Br-CAMP+MPA). $\mathbf{K}$ Immunofluorescence was performed to analyze the morphological transformation of hESCs.

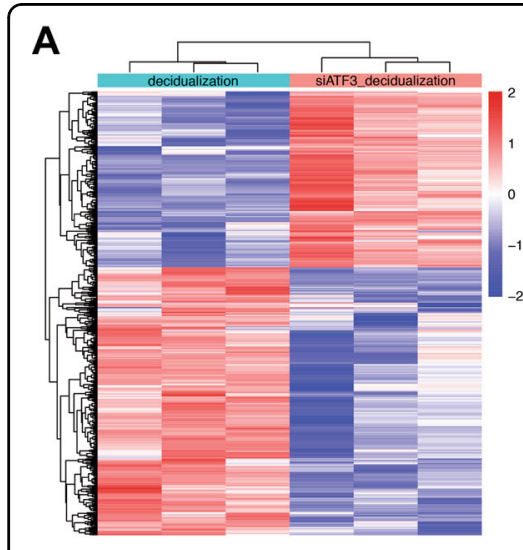

D

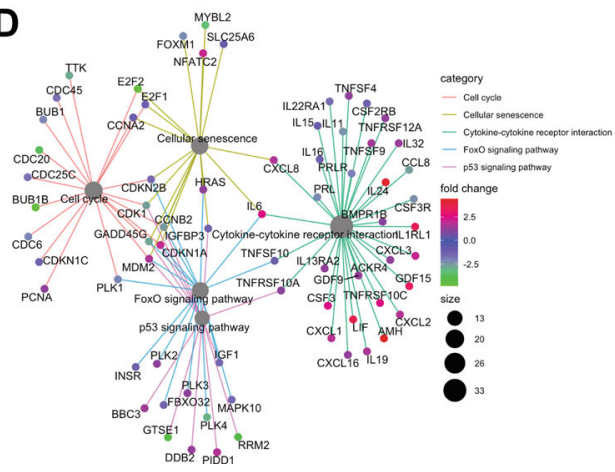

\section{B}

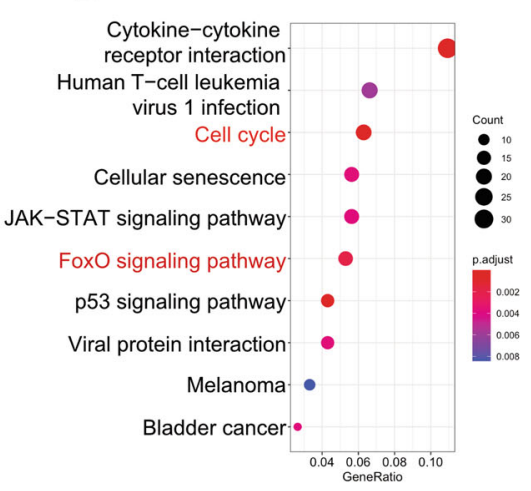

E

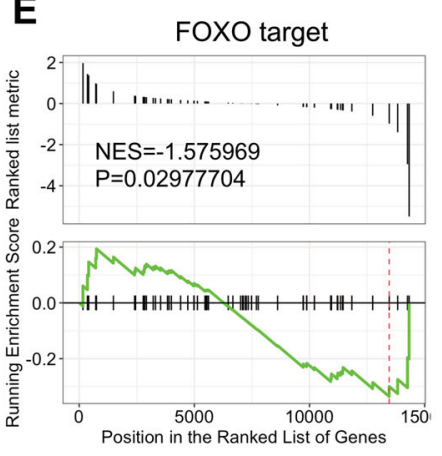

C
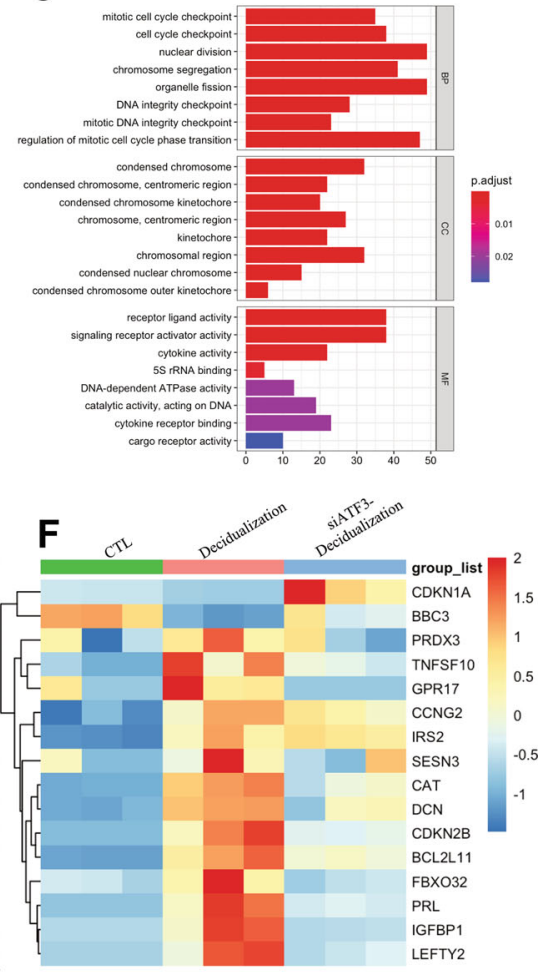

Fig. 3 DEGs in hESCs after knockdown of ATF3 during in vitro decidualization. A Heat map showing hierarchical clustering of gene expression in decidualization and knockdown of the ATF3 group. B, C KEGG pathway enrichment analysis and GO analysis of the DEGs. D The DEGs related to the altered KEGG pathways. E GSEA results showed that the FoxO-targeted gene term was downregulated when ATF3 was silenced. F The relative expression of FoxO1 target genes, such as PRL, IGFBP-1, DCN, and LEFTY2.

\section{ATF3 increases PRL through transcriptionally independent regulation of FOXO1}

RNA-seq analysis was performed to identify genes that are regulated by ATF3 during in vitro decidualization. As illustrated in Fig. 3A, there were 689 differentially expressed genes with 274 upregulated and 415 downregulated when ATF3 was silenced compared with those in the siCTL group during decidualization. In addition, decidualization marker genes, such as PRL,
IGFBP-1, and LEFTY2, were significantly downregulated in the siATF3 group (Fig. 3D, F). To explore the mechanism by which ATF3 regulates decidualization, we further performed KEGG enrichment analysis based on the differentially expressed genes (DEGs). Interestingly, as one of the pathways that is affected most by ATF3, the cell cycle and FoxO signaling pathways were suggested to have a significant role (Fig. 3B, C, E, F). 


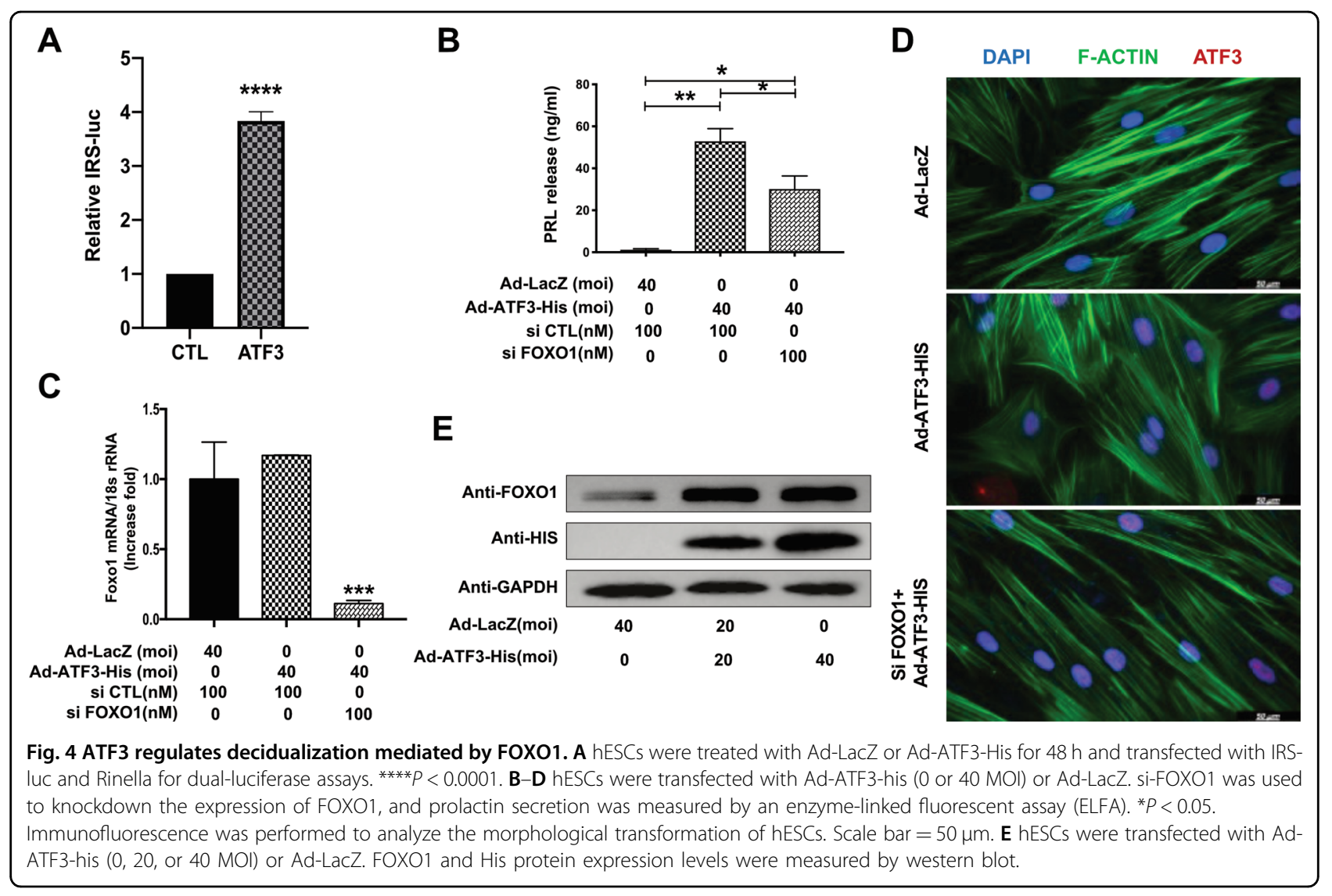

We then performed dual-luciferase reporter assays in hESCs and found that overexpression of ATF3 caused a significant increase in the transcriptional activity of FOXO1 (IRS-luc) (Fig. 4A). FOXO1 is important for the induction of differentiation markers, such as PRL, IGFBP1 , and LEFTY2, in decidual cells ${ }^{22}$. Therefore, we postulated that FOXO1 contributes to the ATF3-induced expression of PRL. As shown in Fig. 4B, C, overexpression of ATF3 increased PRL secretion, which was clearly attenuated by FOXO1 knockdown. Knockdown of FOXO1 with siRNA efficiently inhibited cytoskeletal rearrangement (Fig. 4D). Thus, upregulation of PRL expression by ATF3 may be specifically mediated by regulating FOXO1.

Then, we investigated the effect of ATF3 on FOXO1 and found that compared with the control group, adenovirus-mediated overexpression of ATF3 in hESCs resulted in a concentration-dependent increase in FOXO1 protein expression, as determined by western blot analysis (Fig. 4E). However, the mRNA level of FOXO1 showed no significant difference in hESCs with ATF3 knockdown or overexpression (Fig. 4B and Supplemental Fig. S4C). These findings suggest that ATF3 may upregulate the expression of FOXO1 in a transcription-independent manner.
miR-135b mediates the regulation of FOXO1 by ATF3 during decidualization

As shown in Fig. 3B, we also found that overexpression of ATF3 significantly changed the microRNAs in the cancer pathway. Emerging evidence suggests that microRNAs may have a role in decidualization and implantation $^{23,24}$. To investigate whether there is a microRNA involved in ATF3-induced decidualization, we introduced si-Dicer to ATF3-overexpressing cells and found that siDicer attenuated decidualization in cells treated with ATF3 (Fig. 5A). Differential microRNA expression was found between RIF women and fertile women ${ }^{25}$. Through analyzing miRNA-mRNA network, we found that miR$135 \mathrm{~b}$ may be a potent regulator of FOXO1. In addition, the hsa-miR-135b mRNA levels in RIF patients were upregulated compared to those in fertile women (Fig. 5B, C).

We further found a moderately negative correlation of the expression of ATF3 and miR-135b in mid-secretory endometrial samples from women with RIF and fertile controls $(r=0.4389, P=0.0465)$ (Fig. 5D). qPCR and GSEA results showed that ATF3 downregulated miR135b in hESCs (Fig. 5E, F), and ChIP-PCR was performed to confirm that ATF3 could bind to the upstream promoter of miR-135 (Fig. 5H). We constructed a pair of 


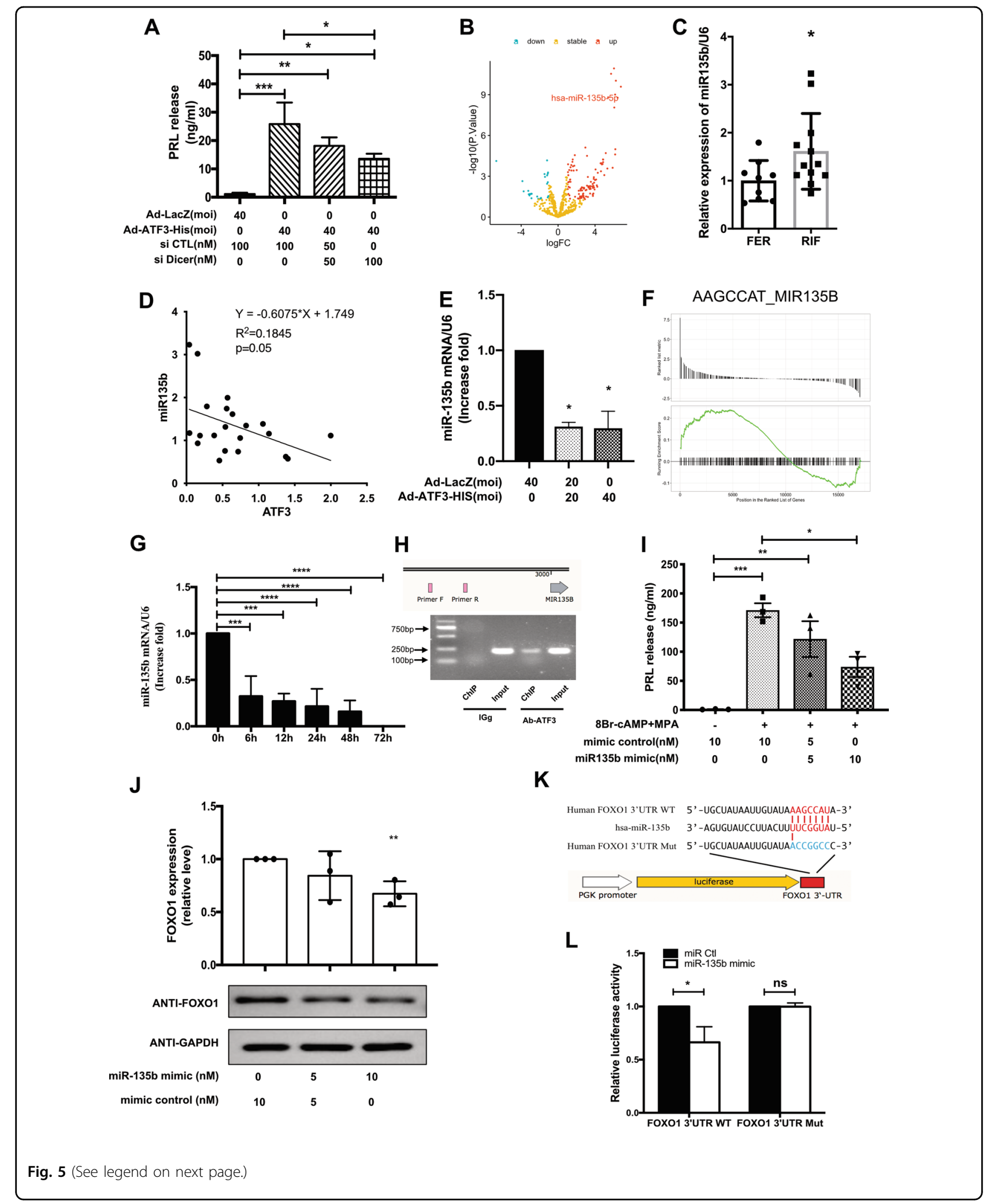

pmirGLO dual-luciferase miRNA target expression vectors that contained the wild-type (FOXO1 3'UTR WT) and mutant target site (FOXO1 $3^{\prime}$ UTR Mut) for the $3^{\prime}$
UTR of FOXO1 separately (Fig. 5K) and found that miR135b significantly suppressed FOXO1 translational efficiency in hESCs (Fig. 5L). The expression of miR-135b in 
(see figure on previous page)

Fig. 5 miR-135b mediates the regulation of FOXO1 by ATF3 during decidualization. A hESCs were transfected with Ad-ATF3-his ( 0 or 40 MOI) or Ad-LacZ. si-Dicer was used to knockdown the expression of Dicer, and prolactin secretion was measured by ELFA. ${ }^{*} P<0.05$, ${ }^{* *} P<0.01$, ${ }^{* * *} P<0.001$. B, C miRNA array and qPCR were performed to detect the differentially expressed miRNAs in RIF patients. Data were downloaded from the GEO database (GSE71332). The expression of miR-135b was validated by qPCR and normalized to U6 expression ( $n=9$ for FER group and 12 for RIF group, $\left.{ }^{*} P<0.05\right)$. D The correlation of ATF3 expression and miR-135b with the data in B. E hESCs were transfected with Ad-ATF3-his $(0,20$, or 40 MOI) or AdLacZ. miR-135b expression levels were analyzed by $q P C R .{ }^{*} P<0.05$. F GSEA results showed that the AAGCCAT miR-135B term was activated after overexpression of ATF3. G hESCs were treated with 8-Br-CAMP+MPA for different times, and then the expression of miR-135b was measured by qPCR. ${ }^{* *} P<0.001,{ }^{* * *} P<0.0001$. H ChIP-PCR was used to detect the binding site of ATF3 on the promotor of miR-135b. I hESCs were transfected with miR-135b mimic $(10 \mathrm{nM})$ or mimic control. After $24 \mathrm{~h}$, the cells were treated with $0.5 \mathrm{mM} 8$-Br-cAMP and $1 \mu \mathrm{M}$ MPA for an additional 3 days. Prolactin released into the medium was measured by ELFA. ${ }^{*} P<0.05$, ${ }^{*} P<0.01$, ${ }^{* *} P<0.001$. J hESCs were transfected with hsa-miR-135b mimic $(0,5$, or $10 \mathrm{nM}$ ) or mimic control. FOXO1 protein levels were analyzed by western blot. ${ }^{*} P<0.01$. K, L Putative 7 bp paired miR-135b/a-target sites in the $3^{\prime}$ UTR of human FOXO1 mRNA. Analysis of miR-135b modulation of the wild-type and mutant FOXO1 $3^{\prime}$ UTR luciferase reporter activity. ${ }^{*} P<0.05$, ns not significant.

hESCs was rapidly downregulated in a time-dependent manner when treated with 8-Br-cAMP and MPA, as determined by quantitative real-time PCR analysis (Fig. 5G). And miR-135b downregulated the protein expression of FOXO1 in hESCs, as determined by western blot analysis (Fig. 5J). Furthermore, miR-135b significantly attenuated decidualization after treated with $8-\mathrm{Br}$-cAMP and MPA (Fig. 5I).

\section{ATF3 regulates the proliferative-secretory transition and thus participates in controlling the WOI in RIF patients}

As mentioned above, DEGs regulated by ATF3 were related to the cell cycle pathway. We analyzed the gene profiles in RIF patients and found that endometrial stromal cell proliferation in RIF patients was hyperactive (Fig. 6A). Immunobiological staining showed that there were more MKI67-positive cells in RIF patients than in controls (Fig. 6B). The expression of CCND1, CDK1, MKI67, PCNA were upregulated, while CDKN1A in endometrium from RIF patients were relatively lower than fertile controls as determined by qPCR (Fig. 6D), and the expression of ATF3 showed a positive relationship with CDKN1A (Fig. 6F). However, during decidualization, the cell cycle was inhibited in stromal cells (NES: $-1.811620, P=5.303467 \mathrm{e}$ $-05)$ which is consistent with the results of ATF3-regulated genes, and overexpression of ATF3 in hESCs caused restrained proliferative activity (Fig. 6G). CDKN1A was downregulated, while CCND1, CDK2, and MKI67 were upregulated by overexpression of ATF3 (Supplemental Fig. S4D). As shown in Fig. 6E and Supplemental Fig. S4E, "TCseq" and "clusterProfiler" packages were used to analyze the genes expression and annotation in control, decidualization, and siATF3-decidualization groups, the results showed that a cluster of genes (cluster 2) that should be upregulated during decidualization was inhibited by siATF3, which were related to FoxO signaling, TGF-beta signaling, and HIF-1 signaling pathway, which are important for decidualization and/or implantation ${ }^{26-28}$. On the other hand, the genes (cluster 1, 6) downregulated during decidualization were upregulated by siATF3, which were enriched in cell cycle, MAPK signaling, and focal adhesion signaling pathway. In the mid-secretory phase endometrium, fewer MKI67-positive stromal cells in the endometrium from fertile control groups compared with RIF patients, and ATF3-positive stromal cells do not express of MKI67 (Fig. $6 \mathrm{C})$. We then analyzed the dynamic genes expression during in vitro decidualization, ATF3 was elevated rapidly, and then genes related to cell proliferation were downregulated and the decidualization related genes were upregulated. (Fig. $6 \mathrm{H})$. Consistent with that, ATF3 was upregulated very early in the secretory phase and decidualization in vitro (Fig. 2F, G); thus, ATF3 may act as a brake of proliferation and a trigger of decidualization during the establishment of the WOI. Since the receptivity window of RIF patients was delayed by 1-3 days, these results suggest that the hyperproliferation of endometrial stromal cells may disrupt the proliferative-secretory transition in RIF patients.

\section{Decidualization is augmented by overexpression of ATF3 in RIF patients}

The above results show that ATF3 had a key role in regulating decidualization and proliferation. We then investigated whether overexpression could augment decidualization in RIF patients. As shown in Fig. 7A, hESCs decidualization from RIF patients was dysregulated, as determined by significantly decreased decidual marker gene expression of PRL. Interestingly, overexpression of ATF3 increased PRL expression and secretion to a level nearly comparable with that of the fertile control (Fig. 7B, C).

We then investigated the mechanism of the augmentation on decidualization by overexpression of ATF3 through RNA-seq. Overexpression of ATF3 resulted in 547 DEGs, with 281 upregulated and 266 downregulated genes (Supplemental Fig. S4A). Overexpression of ATF3 highly regulated the cell cycle, estrogen signaling pathway, cAMP signaling pathway, cytokine-cytokine receptor signaling pathway, and microRNAs in cancer pathways (Supplemental Fig. S4B). 
A

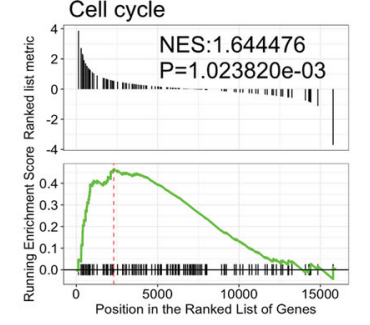

C

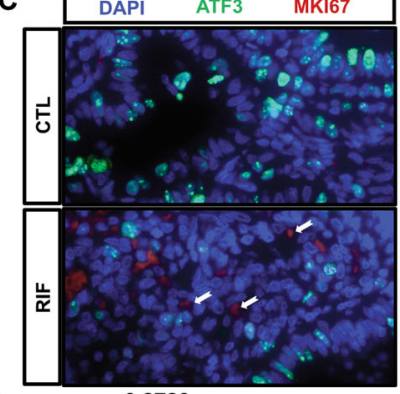

D

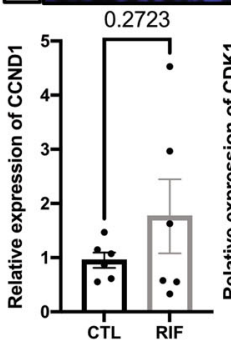

E

E

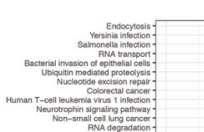

Pathway Enrichment

岩
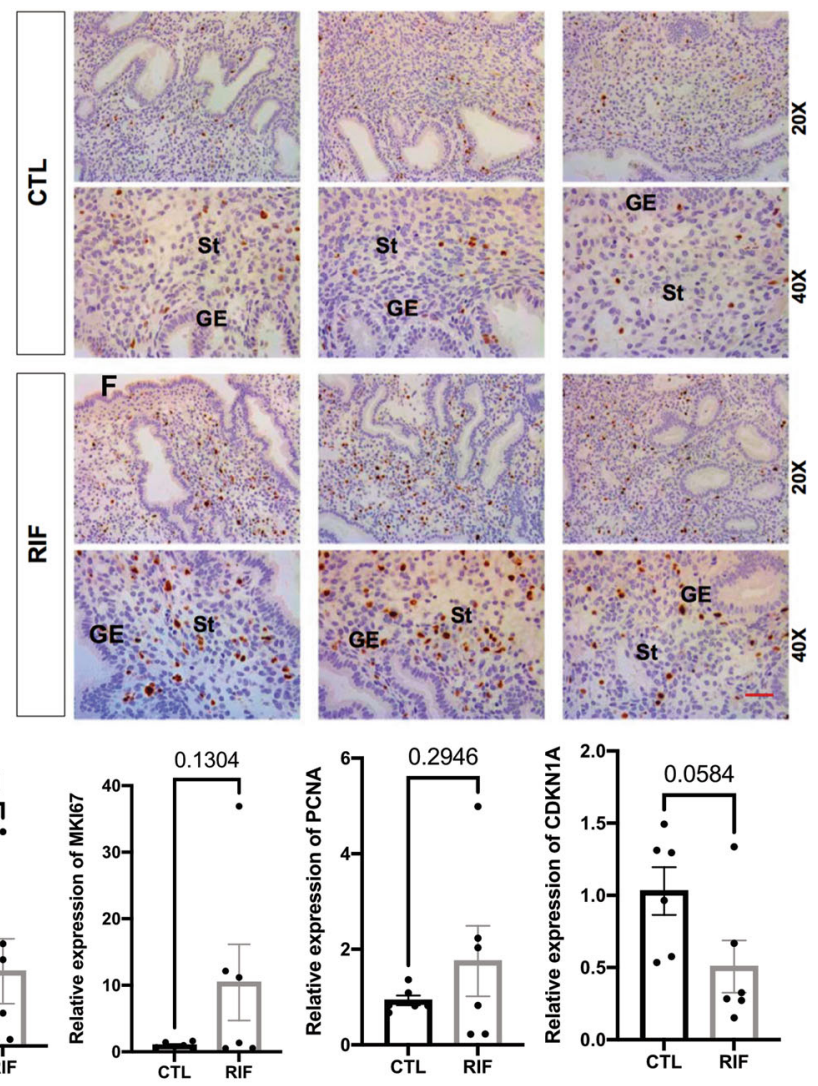

F ${ }^{2.0} 7 \mathrm{Y}=0.7178^{*} \mathrm{X}+0.3206$

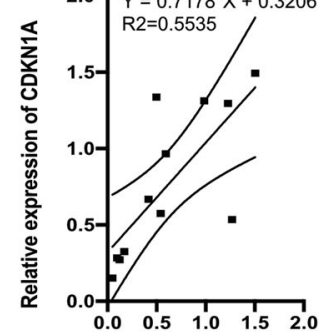

H
Relative expression of ATF3

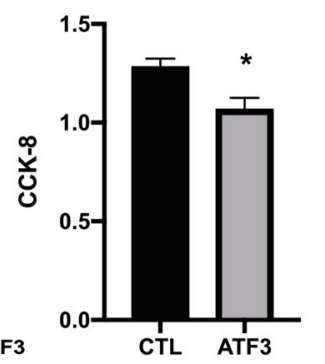

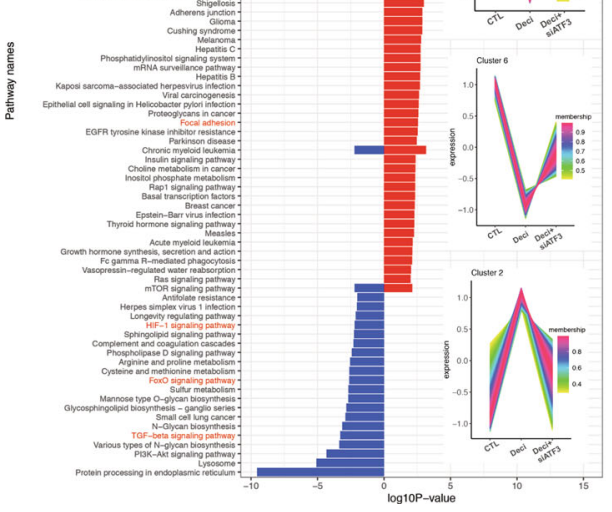

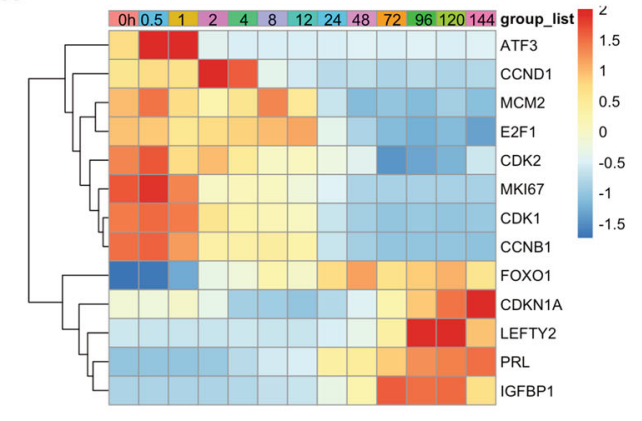

Fig. 6 ATF3 regulates the cell cycle of hESCs. A GSEA results showed that the cell cycle term was activated in the endometrium of RIF patients (NES: 1.644476, $P=1.023820$ - 03). B. MKI67 staining was used to measure the proliferation of stromal cells in the endometrium of RIF patients and fertile controls (ST, stromal cells, GE, glandular epithelium, bar $=50 \mu \mathrm{m}, n=5$ ). C immunofluorescence was performed to determine the expression of ATF3 and MKI67 in endometrium from fertile controls and RIF patients. D The cell cycle-regulated genes were measured by qPCR in the endometrium of RIF patients and fertile controls, $n=6$. E TC-seq analysis was performed to analyze the DEGs in the control, decidualization, and siATF3+decidualization groups. Genes in clusters 1 and 6 were upregulated by siATF3, while those in cluster 2 were downregulated. F The correlation of ATF3 expression and CDKN1A. G hESCs were treated with Ad-ATF3-His or Ad-LacZ, and a CCK-8 assay was performed to detect the cell proliferation rate. $\mathbf{H}$ Genes related to cell proliferation and decidualization change regularly during in vitro-induced decidualization. 


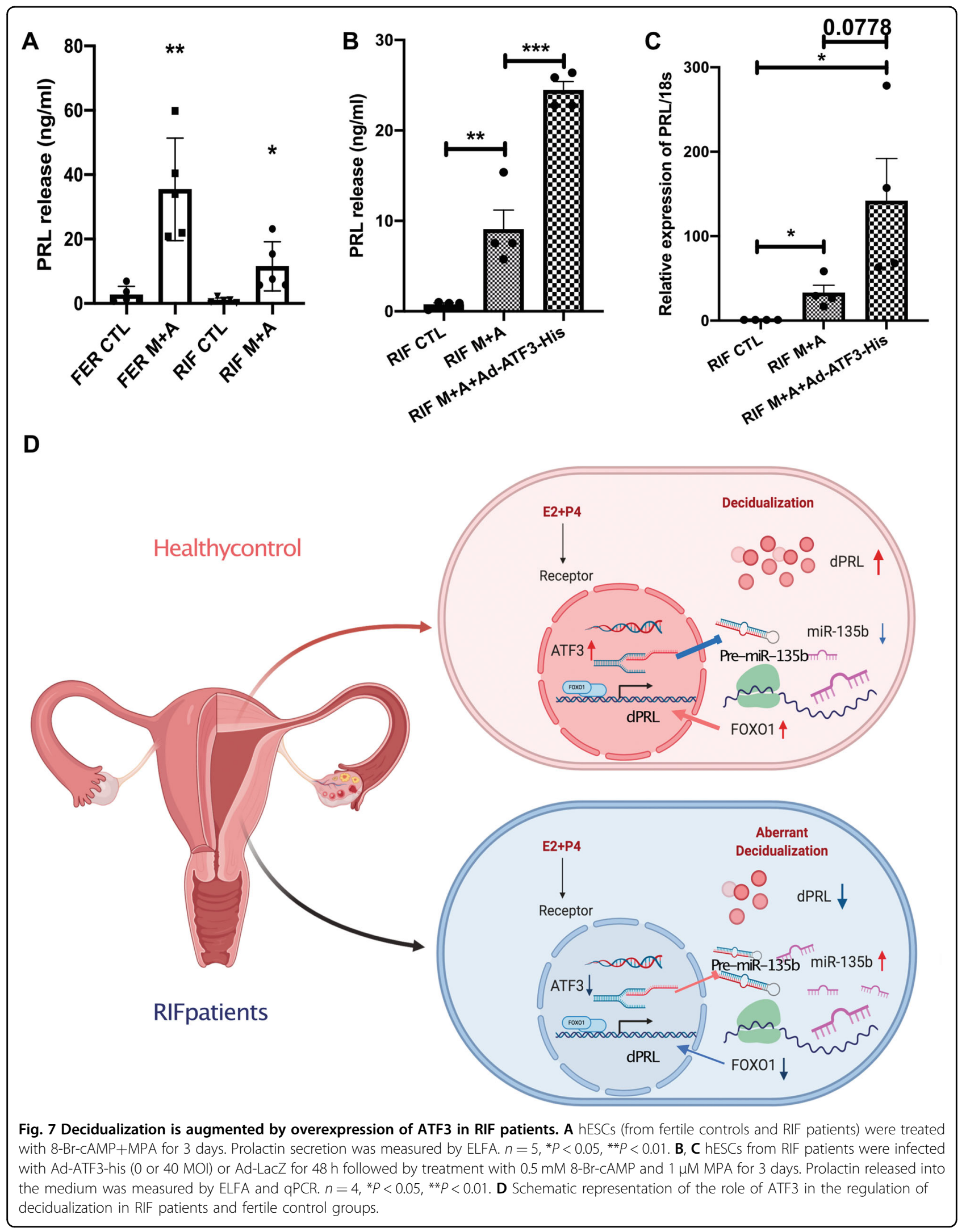




\section{Discussion}

Despite the great progress that has been made in ART over the last 35 years, the embryo implantation rate is nevertheless still not satisfactory, and $\sim 15 \%$ of patients undergoing ART procedures suffer from unexplained RIFs. Recently, a prospective interventional multicenter study found that the receptivity window of RIF patients was delayed by $1-3$ days ${ }^{12}$, suggesting that the acquisition of the endometrial receptivity phenotype is slower in RIF patients than in healthy women. Here, we report that the proliferative-secretory transition was dysregulated in RIF patients and that ATF3 may be a key regulator.

Along with the discovery of new regulatory pathways that control steroid actions, an expectation grew that specific molecular defects in the endometrium would account for RIF or pregnancy loss ${ }^{29,30}$. Thus, there should be some under discovered mechanisms in the maternal uterus $^{31}$. In our previous study, we found that ATF3 was located in both human endometrial epithelial cells and stromal cells, and its expression was dysregulated in RIF patients. In epithelial cells, ATF3 could promote embryo adhesion in vitro by transcriptionally increasing LIF expression $^{32}$. In this study, we focused on the stromal cells to investigate the function of ATF3 on decidualization.

Decidualization is critical and essential for reproduction and involves a series of complex molecular and cellular events to prepare the endometrium for embryo implantation, and dysregulation of decidualization is one of the major causes of $\mathrm{RIF}^{9,10}$. We found that ATF3 was deficient in endometrial stromal cells from RIF patients, and knockdown of ATF3 impaired decidualization, as determined by dysregulated PRL expression and cytoskeletal reconstruction.

ATF3 is an adaptive-response gene that participates in various cellular processes to adapt to extra and/or intracellular changes, where it transduces signals from various receptors to activate or repress gene expression ${ }^{33}$. It has been reported that ATF3 overexpression impacted the proliferation, cell cycle, apoptosis, migration, and invasion of endometrial carcinoma, while estrogen and progesterone had a significant role in its pathogenesis ${ }^{34}$. Our previous study found that estrogen and progesterone could rapidly induce the expression of ATF3 in endometrial epithelial cells, and other studies have also suggested that ATF3 is a downstream gene of estrogen and progesterone $^{32,35,36}$. Here, we found that the expression of ATF3 was increased in the early stage of in vitro decidualization and then decreased, while the expression of ATF3 in the endometrial tissue continued to increase until the mid to late-secretory phase of the menstrual cycle. This may because the 8-Br-cAMP + MPA-induced decidualization protocol could not completely simulate physiological conditions. Recent studies have found that PGE2-induced decidualization is a more physiological model than $8-\mathrm{Br}-$
$\mathrm{cAMP}^{37}$, and that PGE2 has been reported to regulate the expression of ATF ${ }^{38}$. It has been reported that impaired decidualization leads to altered stroma secretion, and disintegrating maternal-embryonic responses, predisposing patients to pregnancy loss ${ }^{39,40}$. PRL is a marker gene of decidualization, and knockout Prl leads to female infertility and lack of embryo implantation ${ }^{41}$. Besides, expression of endometrial prolactin during the "implantation window" was downregulated in patients affected by unexplained infertility and repeated miscarriages ${ }^{42}$. In this study, we found that endometrial stromal cells from RIF patients exhibit a reduced expression of PRL, while supplement with ATF3 could promote the secretion of PRL.

Endometrial decidualization involves the cytoskeletal transformation of stromal cells into secretory decidual cells, during which stromal fibroblasts transdifferentiate into large, epithelioid-like decidual cells. ATF3 is a highly conserved and multifunctional transcription factor that is known for its response to a range of stress signals, and it has been shown that ATF3 links the loss of epithelial polarity to defects in cell differentiation and cytoarchitecture $^{43}$. Genomic and genetic approaches implicate ATF3 as a regulator of cytoskeletal organization and function. Yuan et al. ${ }^{44}$ reported that ATF3 could suppress the metastasis of bladder cancer by regulating gelsolinmediated remodeling of the actin cytoskeleton. Indeed, we found that ATF3 is required for the cytoskeletal organization of endometrial stromal cells and that knockdown of this transcription factor significantly impaired the change in structural features and cell morphology during decidualization.

Currently, plenty of DEGs have been identified during decidualization, implicated in a broad variety of functions, such as the cell cycle, cytoskeletal remodeling, oxidative stress defense, FoxO signaling, modulation of transcription, and chemokine signaling ${ }^{45}$. When ATF3 was knockdown during decidualization, the cytokine-cytokine receptor interaction, FoxO signaling, HIF-1 signaling, and TGF- $\beta$ signaling pathways were downregulated, while the cell cycle, MAPK signaling pathways were upregulated. In addition, overexpression of ATF3 enhances FOXO1 and its target gene expression in a posttranslational regulation. Emerging evidence suggests that microRNAs have a role in decidualization and implantation ${ }^{15,16}$. For example, miR-22, which is upregulated in women with RIF, could impair decidualization by suppressing the expression of Tiam1 and $\operatorname{Rac}^{46}$. Through analyzing differentially expressed miRNAs in the endometrium of RIF patients, we found that miR-135b is a putative regulator of FOXO1, and ATF3 could downregulate the expression of miR$135 \mathrm{~b}$, thus promote the expression of FOXO1 indirectly.

Recently, clinical research found that the WOI of RIF patients may be delayed and that implantation failure could be linked to inadequate timing of embryo transfer ${ }^{12}$. 
In this study, we revealed that the endometrial stromal cells from RIF patients were hyperproliferative, while the cell cycle should be arrested during decidualization, suggesting that the transition from the proliferative to secretory phase was blocked. Furthermore, we found that silencing ATF3 during decidualization activated the cell cycle, while overexpression of ATF3 could limit the proliferation of hESCs. ATF3 is a regulator of the cell cycle in many other tissues or cell lines, e.g., ATF3 can inhibit the expression of AR, MMP2, and AKT, thereby inhibiting cell proliferation and invasion ${ }^{47}$. ATF3 expression is maintained at relatively low levels in quiescent cells. Extensive studies have characterized ATF3 as an adaptiveresponse gene that is induced by a wide variety of signals, including those initiated by cytokines or physiological stresses $^{33}$. We also found that during the menstrual cycle, ATF3 was highly upregulated in the very early-secretory phase, at which point stromal cells stopped proliferating and began to differentiate. Therefore, we hypothesize that ATF3 may disrupt the cell cycle, trigger decidualization, and has a key role in the establishment of the WOI.

In summary, our study found that ATF3 has an important role in decidualization by upregulating FOXO1 via suppression of miR-135b expression and participates in the regulation of the proliferative-secretory phase transition by regulating the cell cycle. Overexpression of ATF3 could augment decidualization in RIF patients. These findings indicate that dysregulation of ATF3 in the endometrium is a novel cause of RIF and may therefore represent a potential therapeutic approach for RIF.

\section{Materials and methods}

\section{Patients and consent to participate}

The patients involved in this study were recruited from the IVF unit of the Reproductive Center of the Affiliated Drum Tower Hospital of Nanjing University Medical School from February 2014 to December 2019. A total of 51 RIF patients and 51 fertile patients were enrolled. Women with hydrosalpinx, endometriosis, or adenomyosis were excluded. The details of these patients are listed in Supplemental Table S1.

\section{Isolation and in vitro decidualization of hESCs}

hESCs were isolated and cultured as previously described $^{48}$. T-hESCs were obtained from ATCC. To induce decidualization, hESCs were cultured with final concentrations of $0.5 \mathrm{mM} 8$-Br-cAMP and $1 \mu \mathrm{M}$ medroxyprogesterone acetate (MPA) (Sigma, St. Louis, MO) in DMEM/F12 (Gibco BRL/Invitrogen, Carlsbad, CA, USA) containing 2.5\% charcoal/dextran-treated FBS (HyClone; Thermo Scientific, South Logan, UT, USA), $100 \mathrm{IU} / \mathrm{ml}$ penicillin, and $100 \mu \mathrm{g} / \mathrm{ml}$ streptomycin. We referred to this hormonal treatment as 8Br-cAMP + MPA. Differentiation was assessed by examination of cell morphology under phase-contrast microscopy at various times during the treatment and by measuring the expression of decidualization-specific marker gene PRL as previously described ${ }^{9}$. For particular treatments, cells were pretreated with Ad-ATF3-His (abm, 071147A), Ad-LacZ, siATF3, or siCtl for 2 days and then treated with 8Br-cAMP and MPA for 3 days.

\section{Immunofluorescence staining for ATF3 and F-actin filaments}

hESCs grown in 24-well plates were treated with siRNA or Adenovirus for 2 days and then exposed to a decidualization stimulus of 8-Br-cAMP plus MPA for 3 days and then fixed with $4 \%$ paraformaldehyde $(w / v)$ for $30 \mathrm{~min}$ at room temperature. Next, the cells were washed with $\mathrm{PBS}$ and permeabilized with $0.5 \%$ Triton $\mathrm{X}-100$ in PBS at room temperature. Subsequently, the cells were blocked with 3\% BSA in PBS and incubated with primary antibodies against ATF3 (1:50, 18655s, CST) at $4{ }^{\circ} \mathrm{C}$ overnight. Next, the cells were washed with PBST and then incubated with Donkey antiMouse IgG $(\mathrm{H}+\mathrm{L})$ Highly Cross-Adsorbed Secondary Antibody, Alexa Fluor 594 (1:100, A-21203, ThermoFisher Scientific), and fluorescein isothiocyanatelabeled phalloidin (1:300; P5282, Sigma, St. Louis, MO, USA) at room temperature. Cell nuclei were stained with DAPI.

\section{Dual-luciferase reporter assay}

The wild type and mutant sequence of $3^{\prime}$ UTR of FOXO1 were cloned into the pmirGLO luciferase reporter plasmid (Promega) and sequenced to confirm the resulting plasmid by GenScript Biotech. hESCs were cultured in 24-well plates and transfected with the indicated plasmids. Cells were harvested, and the luciferase activities were analyzed after $48 \mathrm{~h}$ using a DualLuciferase Assay System (Promega) with a luminescence counter (Berthold Technologies) according to the manufacturer's instructions. For normalization according to the transfection efficiency, firefly luciferase activity was normalized to the corresponding Renilla luciferase activity.

\section{RNA isolation and real-time PCR}

Cells were lysed using TRIzol reagent (Sigma), and total RNA was extracted following the standard manufacturer's protocol. Up to $2 \mu \mathrm{g}$ of total RNA was reverse transcribed in a final volume of $20 \mu \mathrm{l}$ to generate cDNA with $5 \times$ All-InOne RT Master Mix (abm, G492). Real-time PCR analysis was performed with SYBR Green dye and measured on an Analytik Jena instrument to quantify the ATF3, PRL, and FOXO1 mRNA levels. The housekeeping gene 18S rRNA was used as an internal control. The specific primer sequences are listed in Table S2. 


\section{RNA-seq and data analysis}

hESCs were cultured in $6 \mathrm{~cm}$ dishes and transfected with the indicated siRNA for 2 days, then decidualized for another 3 days. Total RNA of these cells was extracted with TRIzol reagent. The transcriptome sequencing and analysis were conducted by OE Biotech Co., Ltd. (Shanghai, China). Clean reads were mapped to reference genome (GRCh38. p12) using hisat2 and DEGs were identified using the DESeq ( $P$-value $<0.05$ and $\mid \log 2$ foldChange $\mid>1$ was set as the threshold for significantly differential expression). KEGG, GO and GSEA was performed using clusterProfiler ${ }^{49}$. And TCseq package ${ }^{50}$ was used to analyze the dynamic gene expression in the $\mathrm{R}$ software ( $\mathrm{R}$ version 4.0.2).

\section{Western blot}

Proteins were extracted as described previously ${ }^{51}$. Equal amounts $(30 \mu \mathrm{g})$ of protein were separated on a $12 \%$ SDSpolyacrylamide gel and transferred onto polyvinylidene fluoride membranes (Millipore, Billerica, MA, USA). Immunoblotting was performed by incubating the membranes with primary antibodies against ATF3 (1:1000; HPA001562, Sigma), FOXO1 (1:2000; Cell Signaling Technology, Danvers, MA, United States), His-Tag (1:2000; M30111, Abmart, Shanghai, China), and GAPDH (1:10,000; AP0063, Bioworld Technology, MN, USA).

\section{Immunohistochemical staining}

Fresh tissues were fixed, embedded in paraffin, and serially sectioned. Antigen retrieval was conducted by autoclaving the samples at $121^{\circ} \mathrm{C}$ for $15 \mathrm{~min}$ in the presence of citrate antigen retrieval solution. The samples were incubated with antibodies against ATF3 (1:600; HPA001562, Sigma) overnight at $4{ }^{\circ} \mathrm{C}$ followed with immunohistochemical staining kits (Zhongshan Golden Bridge). Control sections were run concurrently with the experimental sections using nonspecific rabbit IgG. Nonspecific staining was not detected in the controls. $\mathrm{H}$-score was calculated with IHC-Profiler ${ }^{52}$ and ImageJ software (imagej.nih.gov/ij) based on staining intensity and percentage of positive staining cells, and high positive staining scored 3 , positive staining scored 2, low positive scored staining 1 , and negative staining scored 0 . In this study, we only calcite the staining score of the endometrium stromal cells.

\section{Chromatin immunoprecipitation (ChIP)/PCR assay}

$70 \%$ confluence hESCs were treated with Ad-ATF3-His or Ad-LacZ for 2 days. Crosslink, cell lysis, genomic DNA fragments extraction were performed as described ${ }^{32}$, and then immunoprecipitated using ATF3 antibody (ChIP Grade, ab207434, Abcam) and nonspecific IgG as technical control. The specific primers that were used to amplify the hsa-miR-135b promoter DNA fragments containing a putative ATF3 binding sequence were $5^{\prime}$ -

\section{CAGCTGAAGCCCTCTTTCTG-3 ${ }^{\prime}$ and ${ }^{\prime}$-AGGAGGG TCTGGGTAAAGGA- $3^{\prime}$.}

\section{Statistical analysis}

All of the experiments were performed at least three times. Statistical analyses were performed using Prism version 7 software (GraphPad, LaJolla, CA) or R software. Data represent the mean \pm SEM of biological replicates. Statistical differences in the mean expression values of the two treatment groups were compared using a two-tailed Student's $t$-test. One-way ANOVA was performed for comparisons among more than two groups.

\section{Acknowledgements}

The National Natural Science Foundation of China is gratefully acknowledged.

\section{Author contributions}

Z.W. and G.Y. analyzed the data and wrote the manuscript; J.L., Y.J., and N.K. provided clinical samples and data; Z.W., Y.L., J.L., and R.J. performed molecular work; J.Z., X.Z., and Z.W. performed IF and histological experiments; Z.W. and G. Y. performed transcriptome analyses; G.Y., H.S., and C.L. revised the manuscript; G.Y. and H.S. designed and supervised the study. All authors contributed to the manuscript.

\section{Funding}

This work was supported by the National Key Research and Development Program of China (2018YFC1004401), the National Natural Science Foundation of China (81871165 and 31872846), a grant from Jiangsu Provincial key research and development (BE2018602), and a special grant for principal investigators from the Health Department of Jiangsu Province

(ZDRCA2016070).

\section{Data availability}

The data sets used and/or analyzed during the current study are available from the corresponding author on reasonable request. RNA-sequencing data that support the findings of this study have been deposited in the NCBI BioProject repository (http://www.ncbi.nlm.nih.gov/bioproject/) with accession numbers PRJNA705039 and SRP224538.

\section{Ethics statement}

The use of human tissues was approved by the institutional review board of the Drum Tower Hospital of Nanjing University on the 5th of December 2013 (2013-081-01). Patient consents were received before biopsy, and the study was performed in accordance with the Declaration of Helsinki.

Conflict of interest

The authors declare no competing interests.

Publisher's note

Springer Nature remains neutral with regard to jurisdictional claims in published maps and institutional affiliations.

Supplementary information The online version contains supplementary material available at https://doi.org/10.1038/s41419-021-03679-8.

Received: 23 November 2020 Revised: 25 March 2021 Accepted: 30 March 2021

Published online: 12 April 2021

\footnotetext{
References

1. Wang, H. \& Dey, S. K. Roadmap to embryo implantation: clues from mouse models. Nat. Rev. Genet. 7, 185-199 (2006).
} 
2. Matsumoto, L., Hirota, Y., Saito-Fujita, T., Takeda, N., Tanaka, T., Hiraoka, T. et al. HIF2alpha in the uterine stroma permits embryo invasion and luminal epithelium detachment. J. Clin. Invest. 128, 3186-3197 (2018).

3. European IVFmC, European Society of Human R, Embryology, Calhaz-Jorge, C. De Geyter, C., Kupka, M. S. et al. Assisted reproductive technology in Europe, 2013: results generated from European registers by ESHRE. Hum. Reprod. 32 1957-1973 (2017).

4. Aikawa, S., Kano, K., Inoue, A., Wang, J., Saigusa, D., Nagamatsu, T. et al. Autotaxin-lysophosphatidic acid-LPA3 signaling at the embryo-epithelia boundary controls decidualization pathways. EMBO J. 36, 2146-2160 (2017).

5. Gu, X. W., Yang, Y., Li, T., Chen, Z. C., Fu, T., Pan, J. M. et al. ATP mediates the interaction between human blastocyst and endometrium. Cell Prolif. 53, e12737 (2020).

6. Rytkonen, K. T., Erkenbrack, E. M., Poutanen, M., Elo, L. L. Pavlicev, M. \& Wagner, G. P. Decidualization of human endometrial stromal fibroblasts is a multiphasic process involving distinct transcriptional programs. Reprod. Sci. 26, 323-336 (2019)

7. Critchley, H. O. D., Maybin, J. A., Armstrong, G. M. \& Williams, A. R. W. Physiology of the endometrium and regulation of menstruation. Physiol. Rev. 100, 1149-1179 (2020)

8. Critchley H. O. D., Babayev E., Bulun S. E., Clark S., Garcia-Grau I., Gregersen P. K. et al. Menstruation: science and society. Am. J. Obstet. Gynecol. 223, 624-664 (2020).

9. Huang, C., Jiang, Y., Zhou, J., Yan, Q., Jiang, R., Cheng, X. et al. Increased Kruppel-like factor 12 in recurrent implantation failure impairs endometrial decidualization by repressing Nur77 expression. Reprod. Biol. Endocrinol. 15, 25 (2017).

10. Zhou, Q., Yan, G., Ding, L., Liu, J., Yu, X., Kong, S. et al. EHD1 impairs decidualization by regulating the Wnt4/beta-catenin signaling pathway in recurrent implantation failure. EBioMedicine 50, 343-354 (2019).

11. Paulson, R. J. Introduction: endometrial receptivity: evaluation, induction and inhibition. Fertil. Steril. 111, 609-610 (2019).

12. Haouzi, D., Entezami, F., Torre, A., Innocenti, C., Antoine, Y., Mauries, C. et al. Customized frozen embryo transfer after identification of the receptivity window with a transcriptomic approach improves the implantation and live birth rates in patients with repeated implantation failure. Reprod. Sci. 28, 69-78 (2020).

13. Montminy, M. Transcriptional regulation by cyclic AMP. Annu Rev. Biochem. $\mathbf{6 6}$ 807-822 (1997)

14. $\mathrm{Ku}, \mathrm{H} . \mathrm{C} . \&$ Cheng, C. F. Master regulator activating transcription factor 3 (ATF3) in metabolic homeostasis and cancer. Front. Endocrinol. 11, 556 (2020).

15. Wang, H., Mo, P., Ren, S. \& Yan, C. Activating transcription factor 3 activates p53 by preventing E6-associated protein from binding to E6. J. Biol. Chem. $\mathbf{2 8 5}$ $13201-13210$ (2010).

16. Wang, H., Jiang, M., Cui, H., Chen, M., Buttyan, R., Hayward, S. W. et al. The stress response mediator ATF3 represses androgen signaling by binding the androgen receptor. Mol. Cell Biol. 32, 3190-3202 (2012).

17. Cui, H., Guo, M., Xu, D., Ding, Z. C., Zhou, G., Ding, H. F. et al. The stressresponsive gene ATF3 regulates the histone acetyltransferase Tip60. Nat. Commun. 6, 6752 (2015).

18. Inoue, A., Yoshida, N., Omoto, Y., Oguchi, S., Yamori, T., Kiyama, R. et al. Development of cDNA microarray for expression profiling of estrogenresponsive genes. J. Mol. Endocrinol. 29, 175-192 (2002).

19. Syed, V., Mukherjee, K., Lyons-Weiler, J., Lau, K.-M., Mashima, T., Tsuruo, T. et al. Identification of ATF-3, caveolin-1, DLC-1, and NM23-H2 as putative antitumorigenic, progesterone-regulated genes for ovarian cancer cells by gene profiling. Oncogene 24, 1774-1787 (2005).

20. Wang, W., Vilella, F., Alama, P., Moreno, I., Mignardi, M. Isakova, A. et al. Singlecell transcriptomic atlas of the human endometrium during the menstrua cycle. Nat. Med. 26, 1644-1653 (2020).

21. Jiang, Y., Jiang, R., Cheng, X., Zhang, Q., Hu, Y., Zhang, H. et al. Decreased expression of NR4A nuclear receptors in adenomyosis impairs endometrial decidualization. Mol. Hum. Reprod. 22, 655-668 (2016).

22. Labied, S., Kajihara, T., Madureira, P. A., Fusi, L., Jones, M. C., Higham, J. M. et al. Progestins regulate the expression and activity of the forkhead transcription factor FOXO1 in differentiating human endometrium. Mol. Endocrinol. 20 35-44 (2006).

23. Estella, C., Herrer, I., Moreno-Moya, J. M., Quinonero, A., Martinez, S., Pellicer, A. et al. miRNA signature and Dicer requirement during human endometrial stromal decidualization in vitro. PLOS ONE 7, e41080 (2012).
24. Yan, Q., Yan, G., Zhang, C., Wang, Z., Huang, C., Wang, J. et al. miR-21 reverses impaired decidualization through modulation of KLF12 and NR4A1 expression in human endometrial stromal cellsdagger. Biol. Reprod. 100, 1395-1405 (2019).

25. Revel, A., Achache, H., Stevens, J., Smith, Y. \& Reich, R. MicroRNAs are associated with human embryo implantation defects. Hum. Reprod. 26, 2830-2840 (2011).

26. Yu, X., Gao, C., Dai, C., Yang, F. \& Deng, X. Endometrial injury increases expression of hypoxia-inducible factor and angiogenesis in the endometrium of women with recurrent implantation failure. Reprod. Biomed. Online $\mathbf{3 8}$ 761-767 (2019).

27. Wijayarathna, R. \& de Kretser, D. M. Activins in reproductive biology and beyond. Hum. Reprod. Update 22, 342-357 (2016).

28. Grinius, L., Kessler, C., Schroeder, J. \& Handwerger, S. Forkhead transcription factor FOXO1A is critical for induction of human decidualization. J. Endocrinol. 189, 179-187 (2006).

29. Kim T. H., Yoo J. Y., Choi K. C., Shin J. H., Leach R. E., Fazleabas A. T. et al. Loss of HDAC3 results in nonreceptive endometrium and female infertility. Sci. Transl. Med. 11, eaaf7533 (2019).

30. Guo, F., Si, C., Zhou, M., Wang, J., Zhang, D., Leung, P. C. K. et al. Decreased PECAM1-mediated TGF-beta1 expression in the mid-secretory endometrium in women with recurrent implantation failure. Hum. Reprod. 33, 832-843 (2018).

31. Gellersen, B. \& Brosens, J. Cyclic AMP and progesterone receptor cross-talk in human endometrium: a decidualizing affair. J. Endocrinol. 178, 357-372 (2003).

32. Cheng, X., Liu, J., Shan, H., Sun, L., Huang, C., Yan, Q. et al. Activating transcription factor 3 promotes embryo attachment via up-regulation of leukemia inhibitory factor in vitro. Reprod. Biol. Endocrinol. 15, 42 (2017).

33. Thompson, M. R., Xu, D. \& Williams, B. R. ATF3 transcription factor and its emerging roles in immunity and cancer. J. Mol. Med. 87, 1053-1060 (2009).

34. Wang, F., Li, J., Wang, H., Zhang, F. \& Gao, J. Activating transcription factor 3 inhibits endometrial carcinoma aggressiveness via JunB suppression. Int J. Oncol. 57, 707-720 (2020).

35. Beleza-Meireles, A., Tohonen, V., Soderhall, C., Schwentner, C., Radmayr, C., Kockum, I. et al. Activating transcription factor 3: a hormone responsive gene in the etiology of hypospadias. Eur. J. Endocrinol. 158, 729-739 (2008).

36. Syed, V., Mukherjee, K., Lyons-Weiler, J., Lau, K. M., Mashima, T., Tsuruo, T. et al. Identification of ATF-3, caveolin-1, DLC-1, and NM23-H2 as putative antitumorigenic, progesterone-regulated genes for ovarian cancer cells by gene profiling. Oncogene 24, 1774-1787 (2005)

37. Stadtmauer, D. J. \& Wagner, G. P. Single-cell analysis of prostaglandin E2-induced decidual cell differentiation: does extracellular 8-Br-cAMP cause artifacts? Preprint at https://doi.org/https://www.biorxiv.org/ content/10.1101/2020.09.18.304212v1.full (2020).

38. Subbaramaiah, K., lyengar, N. M., Morrow, M., Elemento, O., Zhou, X. K. \& Dannenberg, A. J. Prostaglandin E(2) down-regulates sirtuin 1 (SIRT1), leading to elevated levels of aromatase, providing insights into the obesity-breast cancer connection. J. Biol. Chem. 294, 361-371 (2019).

39. Huang, C., Sun, H., Wang, Z., Liu, Y., Cheng, X., Liu, J. et al. Increased Kruppel-like factor 12 impairs embryo attachment via downregulation of leukemia inhibitory factor in women with recurrent implantation failure. Cell Death Discov. 4 23 (2018).

40. Yang, Y., Chen, X., Saravelos, S. H., Liu, Y., Huang, J., Zhang J. et al. HOXA-10 and E-cadherin expression in the endometrium of women with recurrent implantation failure and recurrent miscarriage. Fertil. Steril. 107, 136-143.e132 (2017).

41. Bole-Feysot, C., Goffin, V., Edery, M., Binart, N., Kelly, P. A. Prolactin (PRL) and its receptor: actions, signal transduction pathways and phenotypes observed in PRL receptor knockout mice. Endocr. Rev. 19, 225-268 (1998).

42. Garzia, E., Borgato, S., Cozzi, V., Doi, P., Bulfamante, G., Persani, L. et al. Lack of expression of endometrial prolactin in early implantation failure: a pilot study. Hum. Reprod. 19, 1911-1916 (2004)

43. Donohoe, C. D., Csordas, G., Correia, A., Jindra, M., Klein, C., Habermann, B. et al. Atf3 links loss of epithelial polarity to defects in cell differentiation and cytoarchitecture. PLoS Genet. 14, e1007241 (2018).

44. Yuan, X., Yu, L., Li, J., Xie, G., Rong, T., Zhang, L. et al. ATF3 suppresses metastasis of bladder cancer by regulating gelsolin-mediated remodeling of the actin cytoskeleton. Cancer Res. 73, 3625-3637 (2013).

45. Gellersen, B. \& Brosens, J. J. Cyclic decidualization of the human endometrium in reproductive health and failure. Endocr. Rev. 35, 851-905 (2014). 
46. Ma, H. L., Gong, F., Tang, Y., Li, X., Li, X., Yang, X. et al. Inhibition of endometrial Tiam1/Rac1 signals induced by miR-22 up-regulation leads to the failure of embryo implantation during the implantation window in pregnant mice. Biol. Reprod. 92, 152 (2015).

47. Wang, Z., Xu, D., Ding, H. F., Kim, J., Zhang, J., Hai, T. et al. Loss of ATF3 promotes Akt activation and prostate cancer development in a Pten knockout mouse model. Oncogene 34, 4975-4984 (2015).

48. Liu, H., Jiang, Y., Jin, X., Zhu, L., Shen, X., Zhang, Q. et al. CAPN 7 promotes the migration and invasion of human endometrial stromal cell by regulating matrix metalloproteinase 2 activity. Reprod. Biol. Endocrinol. 11, 64 (2013).
49. Yu, G., Wang, L.-G., Han, Y., He, Q.-Y. clusterProfiler: an R package for comparing biological themes among gene clusters. Omics 16, 284-287 (2012).

50. Wu, M. \& Gu, L. TCseq: Time Course Sequencing Data Analysis (2020).

51. Zhang, M., Zhang, Q., Hu, Y., Xu, L., Jiang, Y., Zhang, C. et al. miR-181a increases FoxO1 acetylation and promotes granulosa cell apoptosis via SIRT1 downregulation. Cell Death Dis. 8, e3088 (2017).

52. Varghese, F., Bukhari, A. B., Malhotra, R., De, A. IHC Profiler: an open source plugin for the quantitative evaluation and automated scoring of immunohistochemistry images of human tissue samples. PLOS ONE 9, e96801 (2014) 